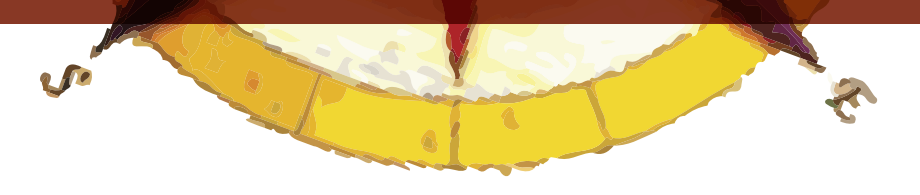

\title{
ESPACIO PÚBLICO INSPIRADOR DE NARRATIVAS URBANAS
}

\author{
Edith Andrea Ortegón Lancheros ${ }^{1}$
}

\section{RESUMEN}

El interés de este trabajo investigativo se centró en la identificación de los espacios públicos significativos para los estudiantes de séptimo grado en la localidad de La Candelaria, teniendo en cuenta la importancia; definida desde la experiencia, los usos y significados que le atribuyen desde sus vivencias personales. Este trabajo es producto de un proceso de investigación que combina dos estrategias; la investigación etnográfica y la enseñanza para la comprensión como modelo que propone la práctica educativa centrada en el desarrollo del proceso de elaboración de significados. La indagación teórica aborda el proceso histórico de construcción del concepto de espacio público, y el uso de la narrativa como categoría más cercana a las ciencias sociales. Para el desarrollo de la propuesta se diseñaron actividades que respondían a la pregunta formativa, construida alrededor de la importancia del espacio público en la construcción de narrativas, dichas actividades están enmarcadas en las fases propuestas por la enseñanza para la comprensión.

Palabras Clave: Espacios públicos, narrativas urbanas, enseñanza para la comprensión.

\section{RESUMO}

O interesse deste trabalho investigativo centra-se na identificação dos espaços públicos significativos para os estudantes de sétimo grau na localidade da Candelaria, tendo em conta a importância; definida a partir da experiência, os usos e significados que designam através de suas vivencias pessoais. Este trabalho é produto de um processo de pesquisa que combina duas estratégias; a pesquisa etnográfica e o ensino para a compreensão como modelo que propõe a prática educativa centrada no desenvolvimento do processo de elaboração de significados. A indagação teórica aborda o processo histórico de construção do concepto de espaço público, e o uso da narrativa como categoria mais cerca ás ciências sociais. Para o desenvolvimento da proposta se desenvolveram atividades que respondiam á pergunta formativa, construída através da importância do espaço público na construção de narrativas, ditas atividades estão sujeitas nas fases propostas pelo ensino para a compreensão.

Palavras Chave: Espaços públicos, narrativas urbanas, ensino para a compreensão.

1 Licenciada en Ciencias Sociales Universidad Pedagógica Nacional. 


\section{ABSTRACT}

The interest of this research is focused on public spaces meaningful identification for seventh grade students in La Candelaria locality, taking account the importance, defined from experience, uses and meanings given from personal experiences. This work is the result of a research process that combines two strategies, ethnographic research and teaching for understanding as model, that propose educational practice focused on the development of elaboration of meaning process. The theoretical research approaches the construction of public space concept as historical process, and use of narrative as a category closer to social sciences. For the development of proposal, were designed activities to respond formative question, around public space importance in narratives construction. This activities are framed in the stages proposed by teaching for understanding.

Keywords: Public spaces, urban narratives, teaching for understanding.

\section{INTRODUCCIÓN}

El ser humano es la única especie capaz de actuar sobre sus condiciones originales de existencia (Montañez, 2009), lo que lo convierte en hacedor de su evolución posterior por medio de la construcción y la transformación de todo lo que le es exterior, que en sus inicios encontrábase sin intervención y ahora se encuentra sujeto a las acciones humanas. No solo es el ser humano quien moldea su entorno; este también lo moldea ya que existen acciones y fenómenos que actúan permanentemente sobre él. Un claro ejemplo de esta relación dual es nuestra ciudad; que constantemente es transformada bajo el afán de la globalización, al ser capital del país debe convertirse en un foco estético atrayente para todas las actividades y ritmos enmarcados en la economía mundial. Es así como sus habitantes también deben transformarse y transformar su estilo de vida; ya no pueden ser simples transeúntes, trabajadores, habitantes, estudiantes, etc. ahora deben ser seres competentes e instruidos que soporten ese acelerado ritmo urbano característico de las ciudades contemporáneas.

En cada época los grupos sociales han consolidado maneras diferentes de construir sus espacios y esas maneras de hacerlo expresan unas relaciones estrechas con las formas de ver y entender el mundo y con las formas de relacionarse con los otros; que están enmarcadas dentro de la interacción con una serie de elementos físicos y materiales que caracterizan dicho espacio. En esta interacción se evidencia la importancia que tienen los espacios de acceso común al presentarse como generadores de procesos identitarios, papel que depende en gran medida de la identificación que se genera a partir de las dinámicas espaciales del ser humano y los roles que desempeñan en la conformación de cada uno de los espacios públicos.

Actualmente, las posibilidades de pensar, teorizar, reflexionar e intervenir sobre las relaciones anteriormente descritas, son diversas y desde el siglo pasado, período que se ha denominado como la era de las metrópolis; caracterizándose por la consolidación de las grandes estructuras urbanas del mundo y el despliegue de teorías sobre la ciudad, asistimos a la intervención de nuevos agentes en la construcción de los escenarios de intereses comunes, que se piensan un nuevo modelo de ciudad y por ende de sociedad.

El proyecto de investigación es una apuesta pedagógica y didáctica que busca identificar la concepción de espacio público que tienen los estudiantes de séptimo grado del Colegio La Candelaria, para generar procesos de enseñanza-aprendizaje y apropiación de los espacios públicos de la locali- 
dad a través de las narrativas urbanas. De este modo, se propone la elaboración e implementación de estrategias didácticas que permitan a través de las narrativas enseñar la categoría de espacio público en la escuela, aventurándose en el estudio de los lugares como elemento para el reconocimiento de las relaciones entre los individuos y sus espacios de vida; desde lo particular porque el lugar procura para cada individuo bienestar y seguridad hasta lo colectivo porque en cuanto a lo social y lo político, permite la creación de identidades urbanas incorporadas por el individuo que establece una apropiación extensiva facilitada por procesos de socialización (Páramo \& Cuervo, 2006).

Los espacios que habitamos se encuentran en permanente construcción gracias a la acción de diversos actores que con modos de andar particularmente atravesados por cosmovisiones personales se congregan dando cuerpo a estructuras espaciales interconectadas, en este sentido es importante poner de manifiesto que dichos mosaicos de lógicas espaciales son permitidos por el desarrollo de un componente cultural, simbólico y regulativo; el código lingüístico. Este es entonces un estudio sobre la importancia del espacio público en la construcción de narrativas urbanas.

Si tenemos en cuenta que las historias del centro histórico de Bogotá; específicamente de La Candelaria, son narraciones constantes sobre los mismos hechos históricos, sobre los mismos personajes y los mismos lugares que conforman el patrimonio histórico de la ciudad; se convierten en relatos únicos y repetitivos que cuentan siempre lo mismo de un lugar. Por ello es primordial destacar la importancia que tienen otros sujetos narradores que también configuran dichos espacios a la hora de construir narrativas desde sus experiencias. Esto significa contar desde otra perspectiva, desde la vida diaria de los estudiantes algunos lugares que han sido contados por una sola voz y desde una sola perspectiva siempre. Los resultados de este estudio resaltan a los estudiantes como actores en el espacio público y como protagonistas de diferentes narrativas propias desde las cuales pueden contar estos espacios.

El interés de este trabajo investigativo se centró en la identificación de los espacios públicos significativos para los estudiantes de grado séptimo en la localidad de La Candelaria, teniendo en cuenta la importancia; definida desde la experiencia, los usos y significados que le atribuyen desde sus vivencias personales.

\section{PRESENTACIÓN: ESPACIO PÚBLICO, INSPIRADOR DE NARRATIVAS URBANAS}

El trazado urbano y las edificaciones del centro histórico de Bogotá, constituyen una parte fundamental del patrimonio cultural colombiano. Las huellas de la historia presentes en este espacio urbano son testimonios del transcurrir del tiempo y de las transformaciones en las ideas y los modos de vida en una ciudad que fue pequeña durante siglos y, de improviso, se transformó en un enorme recinto que alberga hoy en día más de ocho millones de habitantes. "La Candelaria es entonces un conjunto de lugares, eventos, personas, memorias y vestigios" (Candelaria, 1994, p. 10).

En este marco, surge la preocupación por indagar un poco más sobre las transformaciones y nuevas características generadas por los cambios de la ciudad, que construyen otras formas de enunciar o contar el espacio público y la importancia que tiene en la vida social, económica, cultural y política urbana, para consolidar identidades individuales y colectivas. Es por tanto que el tema que nos convoca en este contexto es el espacio público en la localidad de La Candelaria, para ello la pregunta que orientó el proceso de análisis fue ¿Qué importancia tiene el espacio público en la construcción de narrativas urbanas en los estudiantes del curso 702 de la IEDI La Candelaria? y las 
diversas formas de ser contado desde la cotidianidad de los estudiantes.

La Candelaria; Localidad 17, empotrada dentro de otra localidad de mayor extensión denominada Santafé, limita al norte con el Eje Ambiental desarrollado en torno al río San Francisco; que junto con el San Agustín encuadran la ciudad y actualmente corren por canales subterráneos; al sur con la calle cuarta, al occidente la carrera décima y al oriente con la avenida circunvalar y el barrio Egipto. Se caracteriza por ser un terreno notablemente inclinado como consecuencia de su ubicación en las faldas del cerro de Guadalupe. Pero también es una zona donde se sintetiza la heterogeneidad de la sociedad y la cultura colombiana, como se puede describir a continuación:

En sus dominios conviven los más altos dignatarios del Estado con la humilde verdulera de La Concordia; el rico dueño de una casona restaurada se cruza por la calle con el artesano que fabrica guitarras; los estudiantes gritan y pintan con tiza la pared de la casa del poeta Mario Rivero, mientras los teatreros ensayan sus comparsas en el Chorro de Quevedo, ante la mirada embobada del burócrata; ... una marchanta grita a su modo muy especial "iTamales, vendo tamales!" y el aire huele a las deliciosas arepas cuadradas que asa Ligia en plena calle 14; se oye el estampido metálico y amenazante que deja a su paso el "convoy" de guardaespaldas que guarda la espalda del carro veloz de un embajador. Y aquella anciana toma chocolate en la tienda de la esquina... Esta es Colombia resumida en pocas cuadras. (Carranza, 1994, p. 7).

Después del proceso de observación participante en el curso 702, se observaron algunas características que fundamentan el trabajo de investigación; entre ellas, la diversa gama de experiencias y conocimientos que cada uno de los sujetos construye a diario sobre La Candelaria y en particular sobre algunos espacios. Aunque los estudiantes no vivan en esta localidad gran parte de su vida diari se desenvuelve en sus espacio públicos; porqué allí está ubicado el colegio; principal foco de socialización. Las calles a las que les atribuyen un sentido que va más a allá de definirlas como simples sendas, que dan identidad a muchos de ellos porque son espacios que albergan historias de sus cortas pero complejas vidas. Algunos lugares de carácter histórico y emblemático como la Plaza de Bolívar y La Biblioteca Luis Ángel Arango adquieren la connotación de espacios comunitarios, de acceso libre.

Debido a lo anterior, se intenta hacer uso de la narrativa en dos sentidos; primero como estrategia de enseñanza-aprendizaje para abordar el espacio público como concepto geográfico, para asegurar el proceso de comprensión al abordar este tema, y segundo como la forma de visibilizar las experiencias y significados de los estudiantes como actores sociales en los espacios públicos, haciendo más interesante el proceso de enseñanza-aprendizaje porque involucra directamente a los estudiantes y se tienen en cuenta sus contextos en la construcción del aprendizaje.

En algunas clases observadas se evidenció el poco interés y uso de herramientas básicas de comunicación y aprendizaje como la lectura y la escritura; porque siempre se presentaban por parte del docente como actividades aburridas y cuyo aporte era mínimo en el proceso de aprendizaje. Además los estudiantes que se estaban acoplando al proceso de rotación de clases y docentes y al bachillerato como una nueva etapa escolar no tenían en su esquema mental el hábito de lectura, solamente el de escritura, pero ligado a la transcripción de datos. Estas razones definieron la inclusión y el uso de la narrativa como categoría de análisis en el proceso investigativo, con el fin de generar no sólo hábitos sino gusto por leer y escribir; actividades de vital importancia tanto en el ámbito escolar como en toda la vida de un sujeto. Por tanto, se buscó que los estudiantes fueran autores y 
protagonistas de sus propios relatos.

En consecuencia, los objetivos de esta investigación giran en torno a la comprensión de la importancia del espacio público para la construcción de narrativas; para lograr esta meta genérica se plantearon metas específicas como ruta para lograr su alcance; entre ellas: identificar preconceptos geográficos que tenían los estudiantes alrededor del espacio público, demostrar la importancia que tiene este tipo de espacio en la vida social, económica, cultural y política urbana. Proponer las narrativas como una estrategia de enseñanza y caracterizar dichos espacios cercanos a través de las narrativas urbanas para comprender su importancia y significado.

\section{ESPACIO PÚBLICO Y NARRATIVA, ELEMENTOS PARA ENTENDER LA CIUDAD}

Para el desarrollo de las categorías de análisis centrales es necesario partir de algunas definiciones que se han hecho de espacio público y su evolución; con respecto a la narrativa se abordan algunas de sus características y tipos. Para finalizar se aborda la inmersión de ambas categorías de análisis en el aula de clase.

\section{Concepto de espacio público y sus significados}

La interacción entre nuestra especie y el espacio se encuentra en constante evolución y es gracias a ella que en el transcurrir se presentan una serie de hechos de tipo social, económico, político y cultural que se reconfiguran, acomodan, olvidan, fabrican u ocultan con el fin de controlar y regular el papel de los individuos en un colectivo. Por tanto, estudiar la ciudad simboliza estudiar las múltiples historias que han configurado el tejido urbano, valorar a quienes actúan en y sobre ella, las transiciones físicas que ha afrontado a lo largo del tiempo y los significados que se construyen en torno a ella. Por tanto al denotar el carácter de importancia que para este caso posee Bogotá, implica pensar en ella como espacio de simbolización que incide y afecta los procesos de constitución particular de cada individuo.

De esta manera, el espacio público se convierte en uno de tantos lugares sobre los que se tejen las historias de la vida cotidiana y las múltiples experiencias urbanas que dan sentido a la vida de los sujetos en la ciudad. Por tanto:

La impronta sobre la ciudad como escenario de la civilización ha sido determinada por el espacio comprendido ente los límites de lo público y lo privado. Su interrelación ha dado origen, históricamente, a diferentes modelos de ciudad que vinculan ideales políticos, sociales y económicos de las comunidades que lo han habitado (Salazar, 2003, p.6).

Por ello al abordar un estudio sobre la ciudad no se puede hacer distanciando el espacio público, si este es entendido como elemento principal, constitutivo y estructurante del espacio urbano; susceptible de expresarse de varias formas a través de "hábitos, prácticas, rituales, pautas de socialización, manejo del tiempo espacio" (Aguilar, 2001, p. 38).

Al prestar atención a la ciudad desde una perspectiva educativa, donde es agente que promueve el conocimiento a partir de aprendizajes formales e informales en una compleja red educativa de museos, escuelas, universidades, parques, iglesias, calles, entre otros espacios, a su vez que es objeto educativo porque posee un complejo armazón histórico, social y arquitectónico, susceptible de ser 
aprendido; se plantea la posibilidad de difundir los procesos de formación escolar, centrándose en el contexto del que hace parte el educando cotidianamente, lo cual le apuesta a la idea de formar actores sociales éticos y políticos, que encuentren su sentido de pertenecía a un espacio en el que actúan de manera consciente y reflexiva.

El primer acercamiento conceptual que se tiene a la hora de abordar el espacio público, está relacionado con el acceso que todos podemos tener a un espacio físico; es decir su carácter comunitario o lo opuesto a lo privado. Idea que resulta reduccionista porque no se tienen en cuenta otros rasgos característicos de este espacio como sus patrones de uso, su funcionalidad y la identidad que le brinda a la ciudad.

Aunque hay varios autores que coinciden con el aspecto de uso común que tiene el espacio público, consideran que no es la única definición que se puede generalizar sobre esta categoría tan compleja de definir. Lo que si es preciso, es tener en cuenta diversos elementos legales, sociales y culturales que complementan su definición.

Desde lo legal, se considera el Estado como eje central que regula, administra y articula la construcción y protección de este tipo de lugares, lo cual no quiere decir que el espacio público sea de su propiedad. En cuanto a lo social y cultural, Salazar (2003) considera las relaciones que establece la sociedad y que tienen gran influencia estos escenarios, donde se establecen regulaciones procedentes de las costumbres. En este sentido, no se puede entender solamente como un medio físico, soporte de todas las actividades y elementos humanos; por el contrario se debe reconocer el sentido social e histórico de la construcción del espacio. Es así como, se puede explicar su surgimiento, en un contexto social y político específico:

El espacio público fue planteado en términos muy precisos desde su nacimiento, hace aproximadamente 200 años. Fue creado dentro del marco de la burguesía liberal y tiene su sustento ideológico en los ideales de igualdad, libertad y fraternidad, proclamados durante la revolución francesa. Es una noción fundamentalmente moderna. Entenderlo simplemente como un espacio físico es ignorar su historia y privarlo de su carácter transparente y, más exactamente político (Zambrano, 2003, p. 36).

Con lo anterior, es oportuno precisar que para comprender este tipo de lugares se debe tener en cuenta una perspectiva que articule los elementos físicos, sociales y políticos que constituyen el espacio; incluyendo los derechos que tienen los ciudadanos de hacer uso e interactuar en éste. Estos derechos sobre el espacio y su definición legal están consagrados en la Constitución Política de Colombia de 1991; por lo que es más preciso, de acuerdo a Zambrano (2003) hablar de espacio público político. Este tipo de espacio puede ser entendido como un escenario donde los ciudadanos pueden ejercer sus derechos políticos, llevándolos a la práctica como se indica en la legislación.

Los espacios públicos han desempañado papeles estratégicamente significativos en las sucesivas olas de transformación de la ciudad. Durante dicho proceso, el espacio se convierte en un espacio político, en el que se puede visibilizar el ejercicio de los derechos humanos. En este sentido el espacio público se entiende como:

El dominio de todos, como el terreno en donde la ciudadanía se reconoce a sí misma. En el espacio público se establecen regulaciones, provenientes de las costumbres y de las regulaciones 
formales, que permiten y restringen presencias, actividades y significados diversos; lo público es un dominio en el que se ejecutan los ritos de una sociedad: encuentros y desencuentros, intercambios y negociaciones, proclamaciones. En el dominio público, los ciudadanos son -o deben ser- iguales. La ciudad está constituida por los dominios de lo público y lo privado; lo privado es aquello íntimo, interior, familiar, personal (Saldarriaga, 1997).

Esta perspectiva nos permite entender el espacio público como un elemento central de la ciudad, ya que es el escenario donde habitualmente tiene lugar la vida colectiva de sus habitantes; convirtiéndose en un espacio de sociabilidad, encuentro y de representación; donde las relaciones que se establecen entre los sujetos están mediadas por la construcción de significados, de identidades, de roles y de usos, de derechos y deberes. En este sentido, es un espacio de encuentros en el que convergen múltiples experiencias y actividades bajo un sistema de normas, que consolida un "referente imprescindible en la construcción de la identidad de sus habitantes, porque, por un lado, les permite recrear la historia colectiva de la ciudad y por otro, los confronta con sus continuas transformaciones" (Museo de desarrollo urbano [MDU], 2001, p.49).

De acuerdo a lo anterior el espacio público:

Está relacionado esencialmente con la construcción y consolidación de lo político, entendiendo este como el desarrollo de la ciudadanía, las formas de participación y los balances de poder entre los diferentes grupos que habitan la ciudad. El espacio público es el escenario de las luchas sociales que habitan en la ciudad (MDU, 2001, p. 22).

Desde 1990, el espacio público adquiere mayor importancia en el código urbano nacional, por tanto, ya no sólo se considera como el estructurante de la ciudad, sino que pasa a ser el objeto privilegiado del accionar de la administración pública. De esta manera, los andenes, parques, plazas y otros espacios se consideran básicos a la hora de intervenir la ciudad.

Retomando los significados del espacio público, este puede abordarse no solo como un espacio físico, o político; sino desde otras perspectivas que enriquecen su definición. Al hablar de este como un espacio de bienestar, se hace referencia al mejoramiento y adecuación de un medio ambiente para la vida urbana. En este sentido la ciudad debe ofrecer "servicios de saneamiento y de salud adecuados, sistemas de transporte eficientes, espacios para el disfrute del ocio y debe asegurar el desarrollo de las actividades productivas" (MDU, 2001, p. 41).

Como espacio de encuentro, de sociabilidad, de uso y representación:

(...) comprende las diferentes relaciones que establecen los individuos con el espacio físico, con sus significaciones y con las múltiples regulaciones sociales, que aunque no son escritas u obedecen a códigos legales, son el centro de las formas y circulación de la ciudad (MDU, 2001, p. 47).

En este sentido se definen los papeles que juega cada sujeto, se construyen y negocian constantemente reglas de comportamiento y de uso de los espacios, que permiten la convergencia de diversas personas, la realización de actividades diferentes, y la asignación de varios significados en un mismo espacio.

Al abordar el espacio público como un espacio de identidad, este se "constituye como un referente 
imprescindible... porque, por un lado, permite recrear la historia colectiva de la ciudad $y$, por otro, los confronta con sus continuas transformaciones" (MDU, 2001, p. 49). Los diferentes elementos físicos de la ciudad y las costumbres de las personas son referentes de identidad que se construyen y se modifican en cada grupo social, demostrando las expectativas, condiciones de vida específicas de las personas, la forma de representarse a sí mismas y la organización social en cada época.

\section{NARRATIVA ESPACIAL}

El lenguaje entendido como un sistema se signos estructurados que permite la comunicación, puede concebirse como una herramienta que estructura los discursos de un determinado grupo de acuerdo a su contexto espacio-temporal. En este orden de ideas es preciso aclarar que los discursos al ser dependientes del marco social no se expresan de una sola forma y tampoco transmiten un único mensaje, es por ello que "en el discurso, en la manera en que se relata un acontecimiento, se cuenta una historia, en que se construye un punto de vista, se puede encontrar una de las formas elaboradas en que la identidad se expresa y forma al mismo tiempo" (Aguilar, 2001, p. 38).

Aunado a ello, un discurso supone un lenguaje adquirido y practicado socialmente, que es usado por cada sujeto para la interaccion social en un tiempo y en un espacio, por tanto "el lugar no existe sin el elemento de la representacion, la manera en que es construido por las prácticas y los discursos" (Rosaldo, 1991). De esta manera, según Cuervo y Flórez (2004) la narración se inscirbe en el interior del lenguaje y como tal se constituye en una forma de aprender que incide en el desarrollo intelectual, emocional y estético del individuo. Por tanto se le confiere un complejo potencial expresivo que permite describir o dibujar en al mente lo que se explora, observar y comprende.

Resaltando el carácter social de un discurso, la narrativa se constituye en una forma discursiva particular en la que se expresa la subjetividad, el punto de vista, la temporalidad y la espacialidad de las acciones humanas, por tanto:

El punto de vista y la experiencia del actor son cruciales en el uso de las narrativas como instrumento de análisis social ya que proporcionan un acceso directo al mundo de sentido en cual habita la persona. A diferencia de otros acercamientos a la oralidad en las ciencias sociales, el análisis narrativo no busca la "verdad de los hechos" sino acercarse en todo caso a la expresion más fidedigna de la experiencia personal y social (Aguilar, 2001, p. 39).

Dentro de la teoría cognitiva, Bruner (2004) considera la narrativa como pensamiento porque se ocupa de las intenciones y acciones humanas y de los acontecimientos que marcaron su transcurso; a sí mismo, argumenta que contar es un procedimiento opuesto al lógico y lineal, porque permite el surgimiento de lo sorpresivo, anómalo, irregular e inesperado. Así mismo, como categoría de pensamiento "es una forma de razonamiento que estriba en la representación o procedimiento mimético, esto es, que hace uso de los mecanismos de registro, interpretación y construcción que permiten reproducir o representar la realidad" (AMB \& SE, 2007, p. 73).

La narración está inmersa en la manera en que cada persona vive el mundo, es través de un relato que se puede dar cuenta de lo que se conoce y se puede conocer lo desconocido, en tanto, los relatos se convierten en "actos socialmente simbólicos" al adquirir sentido en un contexto social y hacen parte de su construcción. En un sentido amplio, 
El relato puede ser soportado por el lenguaje articulado, oral o escrito, por la imagen, fija o móvil, por el gesto y por la combinación ordenada de todas estas sustancias. $\{\ldots\}$ El relato está presente en todos los tiempos, en todos los lugares, en todas las sociedades (...) es transhistórico, transcultural, el relato está allí, como la vida. (Barthes, 2001, p. 7).

Todos los relatos sin importar su origen, autor o lenguaje cumplen igualmente un papel de significación social, con el que se trata de explicar la realidad social; encaminada a la creación de identidad como seres humanos y como individuos que hacen parte de una sociedad y que deben contribuir a la preservación, conservación y transformación del mundo que habitan. En este sentido, "la construcción de la realidad social no es espontánea y consensual, sino que es el producto de complejas relaciones entre narrativa, poder y cultura. Las relaciones entre actores sociales en los escenarios son entonces tan políticas como sociales" (McEwan \& Egan, 1995, p. 15). Por tanto la construcción social de la realidad conlleva a una lucha entre los diversos modos de fijar y dar sentido, dándole un sentido político.

\section{LA NARRATIVA COMO ESTRATEGIA DE ENSEÑANZA- APRENDIZAJE}

"Los relatos, al igual que la cultura y el lenguaje, han sido constantes compañeros de viaje de los seres humanos, siempre y en todas partes" (White, 1981).

Algunos autores definen la narrativa como "una capacidad humana fundamental" (McEwan et al, p. 9), cuyo papel en la educación debe tener mayor atención, por el enlace de símbolos que trasmite en su lenguaje hilado y por la adopción de un ritmo que surge de las prácticas y actos de las personas, acentuando la relación entre la realidad y la descripción que se relata sobre esta, por ello los relatos son construidos desde la perspectiva de la vida de alguien y dentro de su contexto emocional. Como señala David Lodge "La narrativa es una de las operaciones fundamentales de construcción de sentido que posee la mente; y al parecer es peculiar tanto de los individuos como de la humanidad en su conjunto" (Citado por McEwan et al, p. 11). Al reconocer la narrativa como un rasgo común en toda la especie humana debe tener mayor atención en el ámbito educativo por el vínculo que tiene con las acciones humanas para expresar y construir significados y prácticas.

Una de las principales formas históricas que ha tomado la narrativa es la oralidad, porque se ha consolidado como la forma más usual de comunicación tanto en las culturas netamente orales como en aquellas que desarrollaron la escritura y otros medios de comunicación; razón que hace relevante el papel vital y fundacional que desempeña la narrativa en la enseñanza y el aprendizaje. La narrativa representa una forma primaria, privilegiada e inmanente al discurso; en este sentido, se puede afirmar que: "no debe ser considerada una invención estética utilizada por los artistas para controlar, manipular y ordenar la experiencia, sino como un acto primario de la mente transferido al arte desde la vida" (McEwan et al, p. 4).

Los discursos narrativos se consideran como un elemento fundacional para el aprendizaje, por sus características exploratorias y expresivas. Así, la capacidad de narrar se convierte en una condición del aprendizaje de las formas más elaboradas del pensamiento y la escritura. En este sentido, nadie queda obsoleto para construir un relato, cada persona desde sus intereses es autor de diversas narrativas en toda su vida, lo que sucede es que no se da importancia a los relatos que surgen sino que se consideran como algo esporádico, como un mero elemento de la comunicación. Por ello la importancia de recopilar y darle sentido a las diversas narrativas que construyen los estudiantes 
de grado séptimo del Colegio La Candelaria sobre los espacios públicos que consideran de mayor importancia.

Al involucrar la narrativa como una estrategia de enseñanza-aprendizaje nos permite explorar pensamientos, sentimientos e intenciones de los sujetos sobre un tema específico y materializar en forma de descripción densa los relatos que construyen.

En la educación la narrativa debe abordarse en un sentido amplio, reconsiderando sus diversas formas y funciones; involucrando no sólo su papel de informar e instruir, sino también su uso como forma de conocer, organizar y comunicar experiencias. De esta manera:

El pensamiento narrativo constituye una parte fundamental de nuestra vida cognoscitiva y afectiva; una parte firmemente vinculada con los asuntos éticos y prácticos... es evidente que la narrativa es fundamental para el propósito de comunicar quienes somos, qué hacemos, cómo nos sentimos y por qué debemos seguir cierto curso de acción y no otro. (McEwan et al, p. 17).

En el ámbito escolar el uso de la narrativa es constante, sólo que ha sido limitado a las áreas que tienen una estructura netamente narrativa o las encargadas de la lectura y la escritura; como el español, los idiomas, los estudios literarios y algunos estudios sociales. Aunque en la mayoría de áreas del conocimiento escolar se utiliza la narrativa, es en muy pocas donde se tiene claro su uso, no se da la relevancia requerida a los relatos para hacer un análisis de su contenido, sino que son considerados como una forma en la que estudiantes y docentes se expresan y comunican. Debe tenerse en cuenta entonces que "El hombre es siempre un narrador de historias - nos dice Jean Paul Sartre-; vive rodeado de sus historias y de las ajenas, ve a través de ellas todo lo que le sucede; y trata de vivir su vida como si la contara"(Jackson, 1995, p. 26). Es por ello que en la escuela no deben pasar inadvertidos los relatos, al contrario deben ser analizados y usados como herramienta de enseñanza-aprendizaje por su valor educativo.

Cuando los relatos son creados por los sujetos que hacen parte del proceso educativo escolar-estudiantes y docentes- adquieren mayor importancia porque están narrando y describiendo el contexto o la realidad de la cual hacen parte, estos enriquecen el proceso de enseñanza-aprendizaje porque utiliza unos conocimientos previos que están consolidados para la inmersión de conceptos nuevos que serán comprendidos y utilizados posteriormente en otras situaciones. Es así como "Las historias producen estados de conciencia alterados, nuevas perspectivas, opiniones diferentes, etc.; ayudan a crear nuevos apetitos e intereses; alegran y entristecen, inspiran e instruyen. Los relatos nos ponen en contacto con aspectos de la vida que no conocíamos..." (Jackson, 1995, p. 33). En este sentido se espera que los relatos generen cierto tipo de reflexiones y análisis sobre su contenido y sobre la realidad, para posteriormente transformarla. Para lograr dicho fin, la narrativa debe ser usada no sólo como requerimiento, sino de manera natural y espontánea para que aliada con la enseñanza permita comprender la complejidad de dicha relación.

Para profundizar en la relación entre pedagogía y narrativa, es preciso citar a Angela Moger, quien sostiene que "pedagogía y narrativa coinciden en su confianza en el mecanismo del deseo como modus operandi. La enseñanza y la narración existen, pero duran y funcionan por medio de la postergación, perpetuamente renovada de la satisfacción... La pedagogía, como la narrativa, funciona más por retención que por transmisión..." (Citada por McEwan et al, p. 135), dicha relación se evidencia cuando el maestro o el estudiante se convierte también en narrador. 
Algunos críticos y estudiosos de la educación, que se han interesado por el estudio de la narrativa en contextos sociales y educativos han definido dos áreas en las que la narrativa se ha aplicado. La primera es en la enseñanza de los contenidos, la narrativa se convierte en un elemento estructurante del curriculum. Por otra parte, la narrativa ha sido útil en la investigación educativa, "al representar el objeto de interés del investigador, su método de indagación y la forma que elige para estructurar sus propios escritos" (Gudmundsdottiren, 1995, p. 54).

\section{METODOLOGÍA PARA ABORDAR EL ESPACIO PÚBLICO DESDE LA NARRATIVA}

\section{Enseñanza para la comprensión $(\mathrm{EpC})$}

El enfoque elaborado por Stone, Gardner, Perkins, Perrone, entre otros es un marco fértil para proponer una reorganización de la enseñanza y de los contenidos para que los estudiantes comprendan, es decir, no sólo que puedan conocer determinados datos sino que puedan realizar diferentes acciones en relación con estos. El proyecto de investigación de la Enseñanza para la Comprensión $(\mathrm{EpC})$, se planteó una serie de cuatro preguntas básicas alrededor de las cuales se construyó su marco conceptual. Las preguntas planteadas (Stone, 1998, p. 95) son:

¿Qué tópicos vale la pena comprender?

¿Qué aspectos de esos tópicos deben ser comprendidos?

¿Cómo podemos promover la comprensión?

¿Cómo podemos averiguar lo que comprenden los estudiantes?

Las respuestas a los anteriores interrogantes definen los elementos básicos para el desarrollo de este modelo pedagógico. Con la definición de que vale la pena comprender se identifican los tópicos o temas generativos, y se organizan propuestas curriculares alrededor de ellas; clarificando lo que los estudiantes tienen que comprender articulando metas claras centradas en comprensiones clave; se motiva el aprendizaje de los estudiantes involucrándolos en desempeños de comprensión que exigen que estos apliquen, amplíen y sinteticen lo que saben, y controla y promueve el avance por medio de evaluaciones diagnósticas continuas de sus desempeños, con criterios relacionados directamente con las metas de comprensión (Stone, 1998).

Los tópicos generativos pueden definirse como "cuestiones, conceptos e ideas que proporcionan hondura, significación, conexiones variadas, como para apoyar el desarrollo de comprensiones profundas por parte del estudiante" (Acosta, 2004, p. 242). Los temas generativos se vinculan con las experiencias y las preocupaciones de los estudiantes y pueden abordarse por medio de "una variedad de puntos de acceso desde diversas perspectivas disciplinarias, por medio de múltiples modalidades de aprendizaje o inteligencias, desde diferentes puntos de vista culturales, con una variedad de materiales y recursos de aprendizaje" (Stone, 1998, $\mathrm{p}$. 100). El tópico generativo delinea la materia que será investigada por los estudiantes; por ello debe variar según la edad, los contextos sociales y culturales, los intereses y la formación intelectual de los estudiantes.

Las metas de comprensión, definen lo que se espera que los estudiantes lleguen a comprender; plantea los procesos, ideas, relaciones o preguntas que determinan la ruta de indagación que los 
estudiantes realizarán. Estas metas resultan más útiles cuando se definen de manera explícita y se las exhibe públicamente para que todos los sujetos conozcan que van a realizar durante el proceso de indagación.

Los desempeños de comprensión, pueden definirse como el elemento más importante de la enseñanza para la comprensión; porque "son las actividades que les permiten a los estudiantes entrenarse en el uso de los conocimientos, pasando por diferentes situaciones que le permitirán la comprensión del tema" (Acosta, 2004 , p. 244). Por tanto este elemento se ocupa más de lo que hacen los estudiantes que de lo que hacen los docentes. Los desempeños responden a la pregunta ¿Qué pueden hacer los estudiantes para desarrollar y demostrar su comprensión?, debido a ello, se involucran los estudiantes en la creación de su propia comprensión, en cada etapa de la clase:

La exploración del tema, es el momento inicial de la investigación; donde los estudiantes se acercan al tema generativo y en conjunto con el docente pueden elaborar un balance de lo que saben y lo que les gustaría aprender. Se permite que los estudiantes vean las conexiones existentes entre el tópico generativo y sus propias experiencias e intereses previos. En la investigación guiada, los desempeños que se han propuesto involucran a los estudiantes en la utilización de modalidades de investigación que el docente considera pertinentes para la comprensión de las metas. En rigor, los docentes pueden proponer actividades como la observación, la consulta, la participación y el registro de datos. Las orientaciones que el docente ofrece durante este proceso facilita el aprendizaje desde una comprensión cada vez más compleja. Finalmente, en los proyectos finales de síntesis, los estudiantes demuestran con claridad el dominio que tienen de la meta de comprensión establecida, por ello en este punto se resalta el uso de actividades de socialización o puestas en común.

Aunado a ello, así como lo afirma Stone (1998), los desempeños de comprensión efectivos involucran a los estudiantes en actividades intencionalmente programadas para facilitar el aprendizaje de las metas, están diseñados en secuencias reiterativas, se aprenden por medio de múltiples sentidos y formas de inteligencia, se convierten en un desafío para ampliar la mente y se convierten en un medio de controlar, hacer público y aprender a partir de la comprensión de los estudiantes.

En la evaluación diagnóstica continúa, el análisis de cómo se está avanzando es responsabilidad tanto del docente como de los estudiantes, por ello, la evaluación diagnóstica refuerza a la vez que evalúa el aprendizaje; cada uno aprende analizando el trabajo de sus pares a través de la retroalimentación. "En este proceso se resaltan dos componentes principales: el establecimiento de criterios de evaluación y la realimentación desde el incio hasta la conclusión de la unidad, por medio de diversas fuentes, como el docente, la auto-reflexión y la reflexión de los compañeros" (Acosta, 2004, p. 247). Por eso, la autoevalaución, coevalaución y heteroevaluación recobran sentido y se convierten en oportunidades que le permiten al estudiante reflexionar, criticar y retroalimentarse.

\section{ETNOGRAFÍA EDUCATIVA}

La etnografía se ha consolidado como un método de investigación para identificar, analizar y solucionar múltiples problemas de la educación, desde este tipo de investigación cualitativa, el análisis se focaliza en aspectos dados por los comportamientos de los individuos, de sus relaciones sociales y de las interacciones con el contexto en que se desarrollan.

En este sentido, la investigación pedagógica tiene un marcado carácter social, se centra en la edu- 
cación como proceso complejo, desde una concepción holística, en la que se puede estudiar a los sujetos que intervienen en el proceso educativo, al contexto educativo, al propio proceso o algunos de los componentes que lo integran. Su carácter holístico hace que para cualquier objeto de estudio que se aborde, sea necesario que todos ellos intervengan de una forma u otra en éste; de esta manera se demuestra el carácter etnográfico, en el interés por involucrar a toda la comunidad y hacer propuestas que transformen la realidad existente "sugiriendo alternativas teóricas y prácticas que impliquen una mejor intervención pedagógica" (Nolla, 1997).

En palabras de Rockwell, la esencia de la experiencia etnográfica es transformarnos a nosotros mismos, es decir, transformar nuestras concepciones acerca de otros mundos para producir conocimientos, generando reelaboraciones teóricas, que transformen las concepciones sobre la realidad educativa.

La etnografía educativa se propone como una práctica investigativa que permite analizar las relaciones entre escuela-maestro-estudiante-sociedad, para conocer a fondo los diferentes problemas que se presentan como resultado de la interacción entre ellos, en otras palabras permite entrar a la escuela para conocerla y comprenderla. Por tanto, "este "paradigma alternativo" no acepta la separación de los individuos del contexto, en el cual se realizan sus vidas y por tanto sus comportamientos, ni tampoco acepta ignorar el propio punto de vista de los sujetos investigados, de sus interpretaciones, de las condiciones que deciden sus conductas y de los resultados, como ellos los perciben" (Nolla, 1997, p. 107).

La característica particular de la etnografía educativa es la búsqueda por indagar en los significados de las situaciones cotidianas que se presentan en las escuelas.

Por tal motivo hace énfasis en el estudio micro, en el análisis cualitativo y en la recuperación del sujeto. Para acercarse a la realidad empírica, los investigadores delimitan un universo de estudio, realizan observaciones por periodos prolongados que describen completa y detalladamente, y efectúan entrevistas. Estas técnicas de investigación se emplean simultáneamente pero la observación directa es el medio privilegiado (Piña, 1997, p. 6).

Aunado a ello, el enfoque etnográfico mantiene un proceso de construcción paulatina como metodología de trabajo de campo. En el nivel primer se realizan las observaciones intensivas en el salón de clases con la finalidad de captar y registrar el mayor número de situaciones y acontecimientos. En el segundo momento se plantean las categorías de análisis del trabajo.

\section{CONCLUSIONES}

Se puede afirmar que el espacio público es indispensable en la construcción de narrativas urbanas en los estudiantes del curso 702 de la IEDI La Candelaria, por cuanto se constituye en los lugares más comunes de sus experiencias diarias. Pero son mayormente reconocidos los espacios públicos profanos que al ser abiertos y de libre acceso permiten practicar un sinnúmero de actividades del interés de los estudiantes, se resaltan los parques, algunas calles y plazas como la del Chorro de Quevedo. Con respecto a la importancia de la Plaza de Bolívar que aunque es un lugar abierto sus características históricas permiten clasificarlo como un lugar sagrado, porque le confiere la identidad no sólo a la ciudad sino al país, como parte de la memoria colectiva. Además está rodeada de edificios de valor histórico y cultural; como la Catedral, la Casa de Nariño, el Palacio de Justicia, entre otros. 
Aunque al iniciar el proceso de investigación los estudiantes no reconocían la existencia y uso de normas para usar el espacio público porque lo consideraban de acceso libre y de uso para todo el mundo; sólo asociaban este concepto a los lugares privados. Fue importante ver como ellos mismos durante todo el proceso comprendían la existencia de los patrones de uso de los lugares públicos y asumían su importancia en cada una de las actividades realizadas en cada uno de los lugares. Por ejemplo en la biblioteca Luis Ángel Arango se reconoce la presencia de personal de seguridad y de apoyo en las salas de consulta que recuerdan algunos comportamientos para estar en estos lugares; hacer silencio, no correr en las salas y no jugar en los ascensores; son las conductas más recomendadas.

Involucrar a los estudiantes en procesos investigativos como lo plantea la enseñanza para la comprensión permite que ellos se reconozcan y se sientan como sujetos activos en el proceso de enseñanza-aprendizaje; que puedan aportar, argumentar y construir alrededor de un tema; dinamiza las clases en el aula. Aunado a ello, abordar o utilizar temas del interés de los estudiantes en las clases los motiva porque se hace uso de su cotidianidad para explicar temas académicos. En este sentido el tema que nos convocó en esta investigación: espacio público y narrativas urbanas en la Candelaria, le dio gran protagonismo a los estudiantes que en cada fase del proceso eran autores activos; como lo demuestra la construcción de la crónica en la que convergen las diferentes narrativas construidas por los grupos de trabajo para los espacios públicos de la localidad que son de mayor importancia para ellos.

De esta manera, el currículo comprometió a los estudiantes en un trabajo importante y en un desafío intelectual; donde se vincularon sus preocupaciones, intereses y experiencias en sus contextos específicos. Aunado a ello, en el marco de la pedagogía para la comprensión se logró generar, atraer y retener el respaldo generalizado del trabajo académico riguroso por parte de los estudiantes y docentes.

El modelo pedagógico propuesto permitió evidenciar el desarrollo de la comprensión de los estudiantes, porque no se trabajó simplemente con la acumulación de datos, sino con el aprendizaje de herramientas que pueden utilizar en otros contextos diferentes al educativo y que les aportan a la formación de un pensamiento crítico, reflejado en el planteamiento y resolución de problemas.

Por otra parte, los elementos básicos de la etnografía: observación participante y entrevista semiestructurada, enmarcadas en el trabajo de campo permitieron al investigador construir una interpretación, materializada en una representación coherente de lo que piensan y dicen los sujetos del grupo intervenido, para construir sus propios conceptos. En la descripción etnográfica se privilegiaron las palabras y prácticas en el sentido de la vida, en la cotidianidad de los actores, logrando que el investigador se aproximara a la realidad que estudia para conocerla, interpretarla y describirla para hacerla entendible a quienes no hacen parte de ella.

En este sentido, se resaltan los aportes de la etnografía educativa al proponerse como una práctica investigativa que permite analizar las relaciones entre escuela-maestro-estudiante-sociedad, para conocer a fondo los diferentes problemas que se presentan como resultado de la interacción entre ellos, en otras palabras permite entrar a la escuela para conocerla y comprenderla.

Utilizar y crear diferentes tipos de narrativas permitió explorar la creatividad de los estudiantes y captar su sentido e importancia como sujetos activos que desde sus experiencias de vida cortas pero llenas de matices diferentes pueden contar los espacios no sólo contar sino significar los públicos 
de la Candelaria. Lo que en palabras de Vygotsky, se traduciría a "los significados de las palabras tienen un origen social; y el sistema de signos del lenguaje está empapado de un significado cultural. (Vygotsky, 1995).

Por tanto, la natural conexión entre narración y aprendizaje no debe despreciarse en la escuela, el aula debe verse y entenderse como una forma real y completa de la vida, como un mundo en sí mismo, en el que cada uno de los miembros es un narrador y al mismo tiempo un oyente, que comparte a diario expectativas, sorpresas, historias, fantasías, leyes y costumbres. En este marco, la narrativa se convierte entonces en el vehículo más adecuado tanto para captar la manera en que las personas constituyen su autoconocimiento como para solicitarles que transmitan su sentido personal organizando su experiencia a lo largo de una dimensión temporal o secuencial.

En consecuencia los relatos no son impresiones o fotografías perfectas del mundo, son interpretaciones de este, y como tal reflejan diferentes resultados en los que se expresan las formas de entender el mundo. Este trabajo nos permitió acercarnos a las interpretaciones de los estudiantes a partir de la creación de narrativas que dan cuenta de su realidad, involucrando la importancia que tiene el espacio público en la vida social, económica, cultural y política urbana, para consolidar sus identidades individuales y colectivas.

Al incluir a los estudiantes en procesos de construcción de narrativas, que requieren de ejercicios básicos y constantes de lectura y escritura permitió lograr avances significanticos en el acercamiento, interés y práctica de estas actividades.

\section{BIBLIOGRAFÍA}

Acosta, A. M. (2004). Enseñanza para la comprensión. En M. De Zubiria Samper, Efoques pedagógicos y didácticas contemporáneas. Bogotá: Fundación Internacional de Pedagogía Conceptual Alberto Merani .

Aguilar, M. A. (2001). Narrativas urbanas y sentido de lugar. México, D.F: Universidad de Iztapalapa.

Alcaldía Mayor de Bogota. Secretaría de educación. (2007). Colegios Públicos de excelencia para Bogotá. Orientaciones curriculares para el campo de pensamiento histórico. Bogotá D.C.: Serie Cuadernos de Currículo.

Bogotá., A. M. (2004). "Recorriendo La Candelaria". Bogotá: Secretaria de hacienda.

Candelaria, C. L. (1994). La Candelaria. El centro histórico de Santafe de Bogotá. Bogotá.

Carranza, M. M. (1994). La Candelaria: Historia de una vieja dama. En C. L. Candelaria., La Candelaria. El centro Histórico de Santafe de Bogotá. (pág. 7). Bogotá.

Carreño, J. (16 de Octubre de 2008). Bogotá Paln Centro: Entre Despojos y Sofismas. Desde abajo... la otra posición para leer.

Cobo B, J. G. (2004). Bogotá viva. En V. Asociados, Bogota (págs. 21-23). Bogotá: Villegas editores. 
Colegio La Candelaria. (2009). Pacto de convivencia. Bogotá.

Durán C, M. (2003). Relaciones entre la filosofia y la polis, la metrópolis y la telépolis. En Bogotá y lo Público (págs. 125-131). Bogotá.

Durango, H. (06 de 10 de 2009). Indymedia.org. Recuperado el 21 de 05 de 2011, de http://colombia. indymedia.org/new/2009/10/107336.php

Guber, R. (2001). La etnografia. Método, campo y reflexividad. Bogotá: Norma.

Gudmundsdottiren, S. (1995). La naturaleza narrativa del saber pedagógico sobre 1so contenidos. En McEwan, \& Egan, La narrativa en la enseñanza, el aprendizaje y la investigación (págs. 52-67). Buenos Aires: Amorrortu. editores. S.A.

Habegger, S., \& Mancila, I. (14 de Abril de 2006). areaciega. Recuperado el 25 de Noviembre de 2011, de http://areaciega.net/index.php/plain/Cartografias/car_tac/el-poder-de-la-cartografiasocial

Herrera, J. (s.f.). Cartografia social. Recuperado el 16 de Diciembre de 2011, de http://juanherrera. files.wordpress.com/2008/01/cartografia-social.pdf

Harvey, D. (s.f.). biblioteca virtual clacso. Recuperado el 11 de octubre de 2010, de http://bibliotecavirtual.clacso.org.ar/ar/libros/social/harvey.pdf

Harvey, D. (2003). El Nuevo Imperialismo. Akal.

Jackson, P. (1995). El lugar de la narrativa en la enseñanza. En McEwan, \& Egan, La narrativa en la enseñanza, el aprendizaje y la investigación (págs. 25-50). Buenos Aires: Amorrortu. Editores S.A.

Lindón, A. (1999). Narrativas autobiograficas, memoria y mitos: una aproximación a la acción social. Economía, Sociedad y Territorio , 295-310.

McEwan, H., \& Egan, K. (1995). La narratiava en la enseñanza, el aprensizaje y la investigación. Buenos Aires: Amorrortu editores S.A.

Montañez, G. Geografias y ambientes: Enfoques y perspectivas.

Montenegro, E. (Agosto de 2009). Plan Centro Neoliberal de Bogotá. Prenda Alternativa Periferia .

Museo De desarrollo urbano. (2001). Espacio público por la ciudad. Bogotá: Alcaldía mayor de Bogotá.

Nik, T., Peck, J., \& Neil, B. (Marzo de 2009). SUR Corporación de Estudios sociales y Educacion. Recuperado el 12 de Julio de 2010, de www.sitiossur.cl

Nolla, N. (1997). Etnografía: una alternativa más en la investigación pedagógica. Revista Cubana de Educación Media Superior, 107-115. 
Páramo, P., \& Cuervo, M. (2006). Historia social situada en el espacio público de Bogotá desde su fundación hasta el siglo XIX. Bogotá: Universidad Pedagógica Nacional.

Perkins, D. (1998). ¿Qué es la comprensión? En M. Stone Wiske, La enseñanza para la compresnión. Vinculación entre la invetsigación y la práctica. (págs. 69-92). Buenos Aires: Paidós.

Perrone, V. (1998). ¿Por qué necesitamos una pedagogía de la comprensión? En M. Stone Wiske, La enseñanza para la comprensión. Vinculación entre la investigación y la práctica (págs. 35-68). Buenos Aires: Paidós.

Piña Osorio, J. M. (1997). Consideraciones sobre la etnografia educativa. Perfiles Educativos , 1-22.

Salazar Ferro, C. (2003). Bogotá y lo público. En Bogotá y lo público. (págs. 6-9). Bogotá: Universidad de los Andes.

Stone Wiske, M. (1998). La enseñanza para la comprensión. Vinculacion entre la investigación y la práctica. Buenos Aires: Paidós.

Urbana, D. -S.-D. Observatorio de DINÁMICA URBANA. (Vol. II.).

Witherell, C. (1995). Los paisajes narrativos y la imaginación moral. Tomar la narrativa en serio. En McEwan, \& Egan, La narrativa en la enseñanza, el aprendizaje y la investigación. (págs. 72-85). Buenos Aires : Amorrorte Editores.

Zambrano, F. (2003). La polisemia del espacios público. En Bogotá y lo público (pp. 35-46). Bogotà: Universidad de los Andes. 